\title{
LA LEY N 30886 Y LA NUEVA REGULACIÓN DE LA AUTORIZACIÓN JUDICIAL DE VIAJE DE MENORES.
}

\author{
LAW N 30886 AND THE NEW REGULATION OF THE JUDICIAL TRAVEL \\ AUTHORIZATION OF MINORS.
}

Emilio José Balarezo Reyes*

\section{Resumen}

Con la finalidad de viabilizar el traslado de los menores con sus padres y el adecuado ejercicio de esta práctica se dio la Ley № 30886 la que incorpora el artículo 112 - A al Código de los Niños y Adolescentes, estableciendo las limitaciones y restricciones que se darán al padre que se encuentre en una situación indebida respecto al ejercicio adecuado de la Patria Potestad o ha cometido actos en contra de sus hijos, cuando no ha sido inhabilitado en el ejercicio de esta facultad, dejando en potestad al Juez Especializado para que decida o no su intervención en la aprobación de los viajes que podrá realizar su hijo o el menor bajo su responsabilidad, analizando de esta manera la potestad de ejercicio que éste tenga, sin la intervención del Ministerio Público, primando la celeridad en la toma de decisiones bajo factores que guíen una adecuada decisión que tenga como base el beneficio del menor.

Palabras clave: hijo, viaje, juez, patria potestad, alimentos, fiscal, autorización, deudor alimentario.

\section{Abstract:}

In order to enable the transfer of minors with their parents and the proper exercise of this practice, Law No. 30886 was introduced, which incorporates article 112 - A into the Code of Children and Adolescents, establishing the limitations and restrictions that will be given to the father who is in an undue situation regarding the proper exercise of parental authority when he has not been disqualifying leaving the Specialized Judge in power to decide whether or not to intervene in the approval of the trips that the child or minor may take, analyzing in this way the power of exercise that he has, without the intervention of the Public Ministry, prioritizing the speed of decision making under factors that guide an adequate decision that is based on the benefit of the minor.

Magister en Derecho Civil y Comercial por la Universidad de San Martín de Porres. Abogado en ejercicio. Catedrático de Derecho Civil de la Universidad San Martín de Porres, Universidad Privado del Norte - Filial Lima y la Universidad Científica del SUr. Correo electrónico: milobare@hotmail.com 
Keywords : son, travel, judge, homeland, food, prosecutor, authorization, food debtor.

\section{INTRODUCCIÓN.}

Con fecha 19 de Diciembre del 2017 apareció publicado en el Diario Oficial El Peruano la Ley № 30886: "Ley que incorpora el Artículo 112-A al Código de los Niños y Adolescentes sobre Autorización Especial de Viaje de Menores", la cual, de una primera lectura, nos entrega una serie de figuras y temas que de por sí concitan más de una posición jurídica, y también a un análisis de corte social, transversal y multidisciplinario de parte de todos los que se encuentren vinculados a estos temas y con mucha mayor razón a los operadores jurídicos como a los padres y los que tengan bajo su responsabilidad a los menores, debido a lo delicado de las consecuencias que podrían presentarse como consecuencia de las decisiones que se tomen. En ese sentido, se ha podido apreciar con el transcurso del tiempo que el Código de los Niños y Adolescentes como todo cuerpo legal, necesita de una reexaminación de las instituciones que lo conforman, lo que amerita que existan cambios en su estructura como resultado de los cambios que se dan en la aplicación de los conceptos y de los nuevos planteamientos en el ejercicio de las diversas facultades discrecionales que tienen los padres como las autoridades jurisdiccionales.

Prueba de ello, es la presente ley materia de examinación, pero también, por ejemplo, el Decreto Legislativo № 1297 del 30 de Diciembre de 2016 y su Reglamento aprobado mediante Decreto Supremo № 001-2018 -MIMP publicado en Febrero del año pasado, exactamente el 10 de Febrero de 2018. Todos ellos, de acuerdo a las repercusiones que puedan tener, deben de estar acordes con los Principios que se encuentran tanto dentro de la normatividad nacional-siendo esteel caso del Código delos Niños y Adolescentes - Ley 27337como de forma supletoria y ampliatoria en la normatividad internacional en el caso de la Declaración Internacional sobre los Derechos del Niño, que al ser el Perú país firmante, es perfectamente aplicable sus conceptos e instituciones contenidas dentro del mismo.

El rol decisivo del Juez debe de partir del análisis no solo jurídico en torno a estos temas donde se encuentran inmersos menores de edad, sino de un análisis más general sin perder la visión, ecuanimidad e imparcial que la relevancia de este tipo de temas exige; mucho del éxito de la aplicación de estos cambios dependerá de su comprensión en su totalidad, y los criterios a emplearse en concordancia con los principios que guíen el resultado de la búsqueda del bienestar del menor; por ende, la comprensión de instituciones tan tradicionales como vigentes como son la Patria Potestad, los Alimentos 
sobre todo en lo que respecta a su ejercicio y la intervención de instituciones que ayuden a la toma de decisiones, como es el caso del Ministerio Público, Ministerio de la Mujer y Poblaciones Vulnerables, ente que forma parte del Poder Ejecutivo, juegan un papel más que trascendental en la difusión y en la elaboración técnica de las normas que complementan, y amplían las normas especializadas que se han venido dando de manera gradual dentro del ordenamiento ad hoc para este tipo de temas.

La aparición de nuevas figuras como el Reglamento de Deudores Alimentarios (REDAM), el conocimiento que se tiene sobre el mismo, como se ha venido dando su aplicación, juega un rol relevante en la decisión que se tome respecto a la autorización de los viajes en lo que respecta a sus padres, previo análisis del cumplimiento de un deber fundamental como es el respectivo a los alimentos.

Encontramos así una variopinta reunión de conceptos como de instituciones que tratan de dar una salida a este tipo de temas, netamente especializados, y que ameritan un análisis profundo guiado no solo por la legislación sino también por principios guía nacionales dentro del Código de los Niños y Adolescentes, Código Civil y Normas Especializadas como extranjeros en el caso de la Declaración Universal de los Derechos del Hombre y de forma puntual la Convención sobre los Derechos del Niño y la Declaración Internacional sobre los Derechos del Niño, los cuales nos llevan a un cumplimiento obligatorio infra como supra nacional de la legislación vinculada a este tema. Como lo señala el profesor Alex Plácido en torno a la Convención y su importancia, indica lo siguiente: "El rasgo central y distintivo de la Convención sobre los Derechos del Niño lo constituye la consideración que hace de la infancia, desplazando el enfoque tutelar basado en la idea de niño objeto de control hacia el enfoque de protección, fundado en la concepción del niño sujeto titular de derechos" (Plácido, 2014, p.52)

\section{ANTECEDENTES.}

El Código de los Niños y Adolescentes, Ley № 27337 fue publicado en el Diario Oficial El Peruano el 07 de Octubre del 2000 entrando en vigencia el día siguiente, el 08 de Octubre del citado año; desde esa fecha a la actualidad han transcurrido diecinueve (19) años, tiempo en el cual, el citado texto ha sufrido variaciones con el objetivo de adaptarlo a los cambios como también de otorgarle mayor campo de aplicación ante la coyuntura que se ha venido presentando en lo que respecta a esta materia. En diferentes partes de su articulado han existido cambios y expresamos que se vendrán mucho más debido a lo cambiante de nuestra sociedad como de las múltiples circunstancias 
y los casos que se presentan dentro de esta materia, lo que amerita que se dé una adecuada respuesta legal que desde nuestra posición debe de ser integral, bajo un análisis no solo de la legislación especializada nacional como extranjera sino de la evaluación particular y detallada de cada caso.

Así apreciamos que se encuentran plasmados dentro de esta temática el Principio de Celeridad como figura que se sustenta en la rapidez que se tiene que dar sin descuidar la evaluación de los casos que se presenten con el objetivo de dar una salida oportuna a los diferentes problemas en los cuales están inmersos los menores con el objetivo de buscar el mejor resultado a su favor.

A su vez, encontramos el Principio del Interés Superior del Niño, debido a que por la condición y la naturaleza del menor motiva que todas las instituciones como las personas vinculadas a ellos realicen todas las actividades que les corresponda en búsqueda del beneficio del menor. Respecto a este Principio el Dr. Plácido plantea lo siguiente:

...la Convención sobre los Derechos del Niño exige fijar normas para el funcionamiento de las instituciones, los servicios y los establecimientos para niños, y el Estado deberá garantizar su cumplimiento mediante una supervisión y control adecuados. La disposición no se refiere exclusivamente a las instituciones, los servicios y los establecimientos estatales, sino también aquellos que son responsables del cuidado y protección del niño. (Plácido, 2015, p. 230)

La temática que es motivo de reflexión y análisis se encuentra dentro del Código de los Niños y Adolescentes, en el Libro Tercero del citado Código, en el Título I - La Familia y los Adultos Responsables de los Niños y Adolescentes, de forma específica en el Capítulo VIII que es la parte que correspondiente a las Autorizaciones la cual originalmente constaba de dos artículos el $111^{\circ}$ y $112^{\circ}$ que versaban sobre las autorizaciones de viaje tanto notarial como judicial, lo cual permaneció de esa manera hasta la fecha en que se produjo la inclusión del artículo $112^{\circ}-$ A.

En lo que respecta a la autorización notarial de viaje de menores, el mismo tiene sus propias características y procedimiento como requerimientos a seguir dentro del ámbito de la Ley de Notariado y la de Asuntos No Contenciosos de Competencia Notarial con la finalidad de obtener la respectiva Certificación Notarial para el permiso de viajes ya sea dentro o fuera del país.

Lo primero que hay que dejar constancia es que el ámbito de análisis 
temático es el de la intervención judicial para la autorización de viaje de menores. Como es de conocimiento, el rol del juez como operador jurídico trae consigo un grado de preparación y capacitación constante. En el presente caso nos encontramos frente a un Juez Especializado, es decir, un juez que tiene un conocimiento profundo de la materia en la que administra justicia, agregándole a este tipo de materia un criterio mayor que la experiencia como la realidad que cada situación presente le lleve a la toma de una determinada decisión como postura para la solución de este tipo de casos.

Hay que dejar en claro también que no estamos frente a una modificación estructural del artículo $112^{\circ}$ el cual permanece vigente dentro del texto legal sino que lo acontecido es la incorporación de un inciso al ya citado artículo, el cual como veremos incluye figuras y procedimientos que no están contemplados en el texto original y primigenio del citado artículo $112^{\circ}$ que originalmente se mantiene y es el siguiente:

Artículo 112º Judicial.- Es competencia del juez especializado autorizar el viaje de niños o adolescentes dentro del país cuando falten ambos padres, y fuera del país por ausencia o disentimiento de uno de ellos, para lo cual el responsable presentará los documentos justificatorios de la petición. En caso de disentimiento de uno de los padres o de existir oposición al viaje, se abrirá el incidente a prueba y en el término de dos días resolverá el juez, previa opinión fiscal.

La oposición que formule alguno de los padres se inscribirá en el Libro de Oposición de Viaje de los Juzgados Especializados, el que caduca al año.

De un primer contacto con el presente artículo se aprecia en el mismo la intervención del Juez Especializado como operador jurídico decisorio para el permiso de viaje del menor dentro del país situación que se puede presentar en caso los dos hayan fallecido o se encuentren los dos como no habidos y el menor a su vez no se encuentre bajo el cuidado de tutor alguno.

Respecto a este artículo el profesor Chunga Lamonja lo sintetiza de la siguiente manera: "Señala que cuando los padres no ejerzan conjuntamente la Patria Potestad, es competente para otorgar la autorización de viaje el Juez Especializado". (Chunga, 2016, p. 323)

Un segundo punto es la situación que se presenta cuando el viaje se da hacia el exterior; en este caso se dan dos posibilidades o situaciones: la primera, que no se encuentren uno de los dos padres para dar la autorización 
respectiva ya sea porque está fallecido, no habido o en situación que impida ejercer su rol como padre ante esta decisión, para lo cual, el otro padre o la persona que tenga bajo su cargo al menor, deberá de sustentar documentariamente la viabilidad y la necesidad de la salida del viaje, pudiendo darse como ejemplos: la urgencia de una intervención quirúrgica, o la participación del menor en una determinada competencia internacional en la cual represente al país.

Podemos apreciar la existencia de un tercer tema relevante el cual gira en lo referente al disentimiento entre los padres y la oposición de uno de ellos a la realización del viaje. Debemos de advertir que no son figuras iguales sino que se presentan como resultado de las posibilidades en torno al tiempo, el lugar y la duración del viaje en donde los padres no se ponen de acuerdo, por ejemplo, en el caso se dé una tenencia compartida o con un régimen de visitas y por la edad del menor uno de los padres no está de acuerdo con el viaje debido a que su salud por el tiempo que durará el mismo será contraproducente para el menor. No se opone al viaje sino que considera excesivo el tiempo de su duración.

En cambio, la oposición nos expresa la negativa tajante del viaje por parte de uno de los padres desde el inicio de las tratativas, debiendo sustentar las mismas, con el objetivo de no caer en una situación que perjudique al menor, pudiendo ser por ejemplo: una supuesta salida del país por fines médicos pero en realidad es una situación que esconde sacar al menor con el objetivo de que no regrese junto a su padre o su madre, motivado por una situación enfermiza y predeterminada del otro padre o madre con el fin de tener el control total sobre el menor.

Hay un aspecto procesal en esta parte del artículo que es necesario analizar la cual comienza con la institución del incidente, que de acuerdo al profesor Alfaro, lo conceptualiza de la siguiente manera: "En lo forense, cuestión accesoria de un litigio, relacionada con la principal, que se decide separadamente de esta" (Alfaro, 2002, p.473); el cual ha surgido como consecuencia de la oposición de uno de los padres al viaje del menor. Es de conocimiento dentro del ámbito del derecho que esta figura se caracteriza por ser una cuestión que se decide aparte del principal, pero que va vinculado al mismo, e incide sobre el tema principal. En este caso, es un incidente a prueba, consistente en demostrar con los elementos probatorios necesarios que la oposición al viaje está debidamente justificada.

La premura de su solución, ya que se otorga dos días para la toma de decisión del Juez, responde a la protección como también a la búsqueda sin 
dilación alguna de la mejor opción en favor del menor. La intervención del Ministerio Publico, responde a que como ente que vela por la legalidad y la protección de la sociedad, de forma particular la familia y cada una de las personas que la forman, se encuentran respaldados y amparados por ella. De acuerdo al maestro Chunga Lamonja, manifiesta una posición particular frente a este tema cuando indica lo siguiente: "Considero que el término es muy breve, en virtud de que el Juez debe estudiar con mucha cautela si procede o no procede la autorización al viaje." (Chunga, 2001, p.368)

El presente artículo culmina con la mención a una institución inmersa en este tipo de temas vinculados a los menores, como es el Libro de Oposición de Viaje de los Juzgados Especializados, justifica su presencia porque a través de él se desarrolla un registro de cada una de las situaciones donde se ha presentado la Oposición ya que la misma contrae una serie de características como su procedencia, su justificación como también su probanza; lo que se busca es tener antecedentes, y cómo fue resuelto el mismo; por último, se le otorga el plazo de un año de vigencia debido a que sobre el propio menor se pueden presentar posteriores oposiciones como también nuevas situaciones que se produzcan en torno al menor respecto a su traslado ya sea al interior como al exterior, lo que siempre concitara un tema de reflexión y decisión merituada.

Como lo indica en torno al ámbito procesal que se aplica para el permiso de viaje de menores, el doctor Aguilar indica lo siguiente: "Si bien es cierto no se menciona expresamente en nuestra normativa, se debe tener en cuenta que la solicitud de autorización de permiso de salida de menores se realiza ante la vía procedimental del proceso no contencioso regulado en el artículo 749 del Código Procesal Civil." (Aguilar, 2019, p.578)

\section{ANÁlISIS DE LA LEY № 30886: “LEY QUE INCORPORA EL ARTÍCULO 112-A AL CÓDIGO DE LOS NIÑOS Y ADOLESCENTES SOBRE AUTORIZACIÓN ESPECIAL DE VIAJE DE MENORES"}

Artículo 112-A.- Autorización especial de viaje de menores

Cuando uno de los padres se encuentre inscrito en el Registro de Deudores Alimentarios Morosos (REDAM) creado por la Ley 28970, o haya sido condenado con sentencia firme por delito doloso cometido en agravio de sus hijos o en perjuicio de los mismos, sin que se le haya inhabilitado para el ejercicio de la patria potestad, el responsable presentará ante el juzgado correspondiente esta situación a fin de 
que el juez decida en el plazo máximo de dos días la salida del niño o adolescente sin abrir incidente a prueba ni solicitar la opinión del fiscal, salvo casos excepcionales. El supuesto anterior no será aplicable cuando el deudor alimentario moroso haya cancelado la deuda total de la asistencia alimentaria.

El inciso 112-A fue incorporado mediante Ley № 30886 de fecha 19 de diciembre del 2018, al Código de los Niños y Adolescentes. De un primer contacto con el texto de la ley, el cual contiene el artículo que ha sido agregado, se puede apreciar la presencia de otra figura relativamente nueva en nuestro sistema jurídico para los temas vinculados al rubro de la familia como al de menores, y nos referimos al Registro de Deudores Alimentarios (REDAM), el cual dentro de sus múltiples justificaciones para su inclusión y pertinencia dentro del ámbito normativo, es llevar a cabo un registro como también un seguimiento de todos aquellos padres que no cumplan con el compromiso que tienen y aceptaron periódicamente cumplir con el depósito de dinero o de proveer los recursos como implementos necesarios con la finalidad de que el menor pueda desarrollar con normalidad su vida y se le pueda proveer de lo necesario para que lo haga de forma regular.

Como lo plantea el Dictamen de la Comisión de Justicia: “Mediante Ley № 28970 de fecha 12 de enero de 2007, se crea el REDAM el cual se encuentra a cargo del Poder Judicial y donde figuran las personas que adeudan tres (03) cuotas, sucesivas o no, de sus obligaciones alimentarias establecidas en sentencias consentidas o ejecutoriadas, o acuerdos conciliatorios con calidad de cosa juzgada."

Lo que se busca es que a través del control de este tipo de registro se lleve a cabo un seguimiento del cumplimiento por parte del padre que ha sido sentenciado con la obligación de suministrar los alimentos, lo cual de darse el incumplimiento estaría trayendo consigo consecuencias como es la de tener restricciones y limitaciones en su ejercicio de los derechos que como padre este tiene, los cuales pueden ser temporales como también absolutos dependiendo del tiempo del incumplimiento y del comportamiento que el padre tenga, esto de forma general ya que es aplicable para ambos padres.

Una temática relevante y que también constituye una innovación en el tratamiento del presente estudio es la que corresponde a la sanción que se ha dado como consecuencia de que el padre de familia ha concretizado en contra de su hijo un delito doloso o perjuicio, figuras que dentro del ámbito penal no son lo mismo ya que el primero por su naturaleza y concretización por acción u omisión recae sobre el sujeto del menor, en cambio el perjuicio 
vendría hacer el cúmulo de actividades que indirectament? pueden afectar al menor en su integridad psicosomática. Los fundamentos de esta norma en este punto, hace referencia al Delito de Omisión de Asistencia Familiar, debidamente tipificado en el Código Penal, debido a que el Perú presenta un porcentaje de personas que se encuentran dentro de esta situación, muchos de ellos cumpliendo pena efectiva en los centros penitenciarios de nuestro país. Otro rumbo complementario a este tema es el del Maltrato Infantil que un porcentaje importante de menores sufre a diario en nuestro país, así esta figura puede plasmarse de forma física como también psicológica. Hay que indicar que el propio artículo señala que el tema materia de controversia debe ser ventilado como analizado en un proceso penal lo que ha derivado en una sentencia condenatoria firme.

Ahora, esta situación muchas veces no es comunicada a las autoridades con la finalidad de proceder a las limitaciones respectivas en el ejercicio que tiene esta persona respecto de sus derechos como padre por lo que la persona responsable del menor, es decir, quien lo tenga a su cargo, puede utilizar esta figura con la finalidad de evitar que el padre sancionado penalmente impida u obstaculice la salida del menor del país, ya que este se encontraría en una situación en la que no podría intervenir. Este tipo de situaciones se darían en el caso de que los padres a cambio del permiso de viaje chantajearan a los hijos y a las personas responsables de los mismos con la finalidad de dar su consentimiento para el viaje del menor.

En la parte correspondiente a la tramitación, es importante señalar que existe de manera evidente la presencia de la celeridad debido a que comparando con el artículo 112 se aprecia que no se toma en consideración la opinión del fiscal, ni se forma incidente alguno por lo que todo quedará en manos del Juez Especializado. Termina esta parte del artículo haciendo hincapié a casos excepcionales. Creemos que este tipo de situaciones deben ser debidamente merituadas debido sobre todo a que de proceder la formación del incidente perdería la celeridad requerida para este tipo de casos; tomando aun la condición legal de uno de los padres, podemos encuadrar dentro de estas situaciones especiales una situación de enfermedad que podría ser contagiosa que haga imposible el movimiento del menor.

Se concluye el presente artículo señalando que todo lo anteriormente analizado quedaría sin efecto siempre y cuando el progenitor declarado como deudor moroso llevará a cabo el pago efectivo de toda la deuda que ha tenido acumulada por el concepto de alimentos, lo cual indicaría su predisposición de dar solución a su situación de morosidad por lo que recuperaría sus derechos y facultades sobre el menor. 


\section{CONCLUSIONES}

1. La inclusión del inciso A dentro del Artículo 112 del Código de los Niños y Adolescentes implica la presencia de un tratamiento especializado a una realidad que afronta el menor dentro de la sociedad como es la obtención del permiso para la realización de viajes por parte de sus padres, cuando uno de ellos no se encuentra en una situación idónea jurídicamente, para el ejercicio de las facultades que le son reconocidos.

2. La presencia de figuras modernas dentro del ámbito jurídico institucional como el Registro de Deudores Morosos (REDAM) en lo que respecta a la temática de los menores y adolescentes ayuda a discernir adecuadamente en torno a los mismos respecto a la idoneidad de los padres en su inclusión en la toma de decisión si es viable o no el viaje.

3. Lo que se busca es implementar una política de prevención y de protección en torno al menor para que no sea manipulado ni maltratado por sus padres al momento de dirimir la viabilidad del viaje, ya que, con la finalidad de otorgar su consentimiento para el mismo, pueden utilizar actividades y conductas inapropiadas contra el menor como es el caso de la manipulación.

4. En el caso de la tramitación como la finalidad del otorgamiento del permiso para el viaje a favor del menor nos da como mensaje de manera sustancial que deben considerarse los Principios Sustantivos a favor del menor, como es el caso de la Celeridad en la tramitación donde este fuere posible debido a las consecuencias o resultados que podrían afectar al menor se diera un retraso que afectaría de modo sustancial los intereses de las personas que lo tienen a su cargo como del propio menor.

5. Vemos de lo desarrollado en el presente texto que la idea guía para el desarrollo de este tipo de temas es una correcta interpretación del Principio del Interés Superior del Niño, el cual en todas las dimensiones en las que fuera aplicable lo que busca es el bienestar del menor en una edad susceptible a sobrexposición, como también al maltrato y a la explotación, pero a su vez no perjudicarlo en la pérdida de oportunidades que se le podrían presentar como consecuencia del retraso como la negación al traslado. 


\section{REFERENCIAS}

Aguilar, J. (2019). Comentario al Artículo 112 del Código de los Niños y Adolescentes. Código de los Niños y Adolescentes Comentado. Jurista Editores.

Alfaro, R. (2002). Diccionario Práctico de Derecho Procesal Civil. Gaceta Jurídica.

Chunga, F. (2016). Comentarios al Código de los Niños y Adolescentes. Infracción penal y los derechos humanos. GRIJLEY.

Chunga, F. (2001). Derecho de Menores. GRIJLEY.

Plácido, A. (2015). Manual de Derechos de los Niños, Niñas y Adolescentes. Instituto Pacifico.

Plácido, A. (2014). El Sistema de responsabilidad por infracciones a la ley penal conforme a la Convención sobre los Derechos del Niño y su aplicación directa por los órganos jurisdiccionales especializados. Propuesta de reforma del Código de los Niños y Adolescentes del Perú. Libro de Ponencias del I Congreso Nacional e Internacional de Derecho de Familia, Niñez y Adolescencia. Graphicomp Impresiones E.I.R.L.

Fecha de recepción: 22 de junio de 2019

Fecha de aceptación: 24 de agosto de 2019 